\title{
COVID-19 research: toxicological input urgently needed!
}

\author{
Charlotte Esser $^{1}\left[\right.$ D Katrin Hochrath ${ }^{1} \cdot$ Tamara Schikowski $^{1} \cdot$ Thomas Haarmann-Stemmann $^{1}$
}

Received: 27 May 2020 / Accepted: 3 June 2020 / Published online: 5 June 2020

(c) Springer-Verlag GmbH Germany, part of Springer Nature 2020

In December 2019, in Wuhan, China, a novel coronavirus was identified. This zoonotic severe acute respiratory syndrome coronavirus (SARS-CoV)-2 has quickly and dynamically spread around the globe, and the WHO officially assessed the coronavirus disease 2019 (COVID-19) as a pandemic in the middle of March 2020. ${ }^{1}$ The response of governments and the scientific and medical community has been very strong since then. Recognizing that only understanding this disease will give the tools to fight it, the EU alone has allocated more than 100 Million Euros to fund SARS-CoV-2- and COVID-19-related research. It seems fair to say that an unprecedented amount of resources is made available nationally and internationally, both for research and medical treatment, and for ameliorating the economic fall-out from counter-measures, especially lock-downs. The urgent interest in understanding the many aspects of the disease and perhaps the pull-effect of the huge financial funding opportunities is evident from the number of papers already published, or submitted to preprint servers. Using the search term COVID-19 finds zero papers in PubMed in 2019 , but about 15,000 papers by middle of May 2020. One will be challenged to find any other medical issue with such an explosion of articles in a few months. Another several thousand manuscripts have been written and are posted on preprint servers, such as medRxiv.org or bioRxiv.org, before they undergo peer-review. There are, however, surprisingly few toxicological studies in this area, and we would like to posit that this does not reflect the important areas where toxicology can and should contribute. A literature search and the authors' own interests immediately identify a number of areas, where toxicological and environmental health issues arise, and such expertise is necessary. First, toxic drug reactions or drug-drug interactions are obvious, and indeed, there are several studies looking at the toxicity of treatment

Charlotte Esser

Charlotte.Esser@iuf-duesseldorf.de

1 IUF-Leibniz Research Institute for Environmental Medicine, Düsseldorf, Germany drugs, such as hydroxychloroquine (first hyped, and now scientifically debunked). The issue of drug-induced toxicity is urgent, given older knowledge about the possible damage of anti-viral drugs, or the effects of ACE2 inhibitors used as anti-obesity drugs on ACE2 expression and thus entrance possibilities of the virus into cells (Boeckmans et al. 2020). Then there are contradictory reports such that smoking is a risk factor for the susceptibility to COVID19, but was also suggested as a prevention. Nicotine (and even smoking) is discussed as an ameliorating factor. Such dangerous paths need the expertise and vigilance of toxicology. The toxic effects of smoking are a long-term area of research for toxicologists, and there are hard-core toxicological mechanisms to be discovered and addressed here, such as the role of oxidative stress, aryl hydrocarbon receptor (AHR) signaling, and latent inflammatory responses. In fact, several studies have reported elevated levels of ACE2, the site of cell entry for SARS-CoV-1 and SARS-CoV-2, in the lower airways of current smokers. Unfortunately, bad science and a rush to flag all kinds of substances as potential cures plague the pandemic literature. Second, we can learn from previous studies on the effects of toxic substances or environment sensing signaling pathways on viral diseases. For instance, the role of the AHR, dioxins and its immunosuppressive and immunostimulating effects on different immune cells in the context of a viral infection can teach many lessons for COVID-19, which toxicologists can extract. For example, recent mechanistic studies on mice infected with various RNA and DNA viruses, including Zika virus, dengue virus, influenza virus A (H1N1), and herpes simplex virus-1, revealed that AHR activation suppresses the production of type I interferons and associated protective immune responses (Yamada et al. 2016). The AHR comes up in other scenarios as well. In a study looking at metabolites in COVID-19 patients versus healthy controls, kynurenine metabolites-potential AHR agonists-were increased. Interestingly, kynurenine and proinflammatory cytokines

\footnotetext{
${ }^{1}$ https://www.who.int/dg/speeches/detail/who-director-general-sopening-remarks-at-the-media-briefing-on-covid-19---11-march-2020 (assessed 20.5.2020).
} 
have been reported to synergistically induce the production of IL-6, a key regulator of the acute phase response and one of the predominating cytokines identified in COVID-19 patients. In fact, inhibition of IL-6 signaling, for instance, by antibodies targeting the IL- 6 receptor, might be a promising strategy to counteract COVID-19-associated cytokine storms. Third, there is the issue of air pollution. It goes in two directions: on the one hand, evidence suggests that due to lock-down measures and less traffic, the amount of air pollution has significantly decreased in some regions, giving great opportunities for epidemiological-toxicological research. However, vice versa, the important question arises, how the presence and extent of air pollution or chemical pollution affect the susceptibility and severity of the disease in individuals or people living in polluted areas. According to the 2019 air quality report of the European Environment Agency, the Lombardy region in Northwestern Italy, which was the European epicenter of COVID-19, ranks among the most air-polluted areas in Europe. An association between air pollution, i.e. particulate matter (PM) and polycyclic aromatic hydrocarbons (PAH), and the severity of COVID19 is conceivable, given the exceptionally high COVID-19 mortality rate of $\sim 12 \%$ in Lombardy and adjacent areas in Northern Italy as compared to $\sim 4.5 \%$ for the rest of country. A possible contribution of high air pollution exposure to disease severity, for instance, due to its pro-inflammatory effects and the respiratory or cardiac damage it causes, was pointed out by Italian researchers, whose data suggest such a link (Fattorini and Regoli 2020) for Northern Italy, or by American and Chinese researchers for California and Wuhan, respectively. Exposure to air pollution could predispose highly exposed populations and put them at higher risk for COVID-19. Interestingly, the adsorption of virus (RNA) to particulate matter $\mathrm{PM}_{10}$, as well as community wastewater might help to monitor the spread of the disease, or even identify new clusters where new outbreaks are imminent. Fourth, from an immunotoxicological point of view, we note that vaccination success may depend on the prevalence of immunosuppressive pollutants. Several years ago, a study in the Faroe Island found that low vaccination efficacy correlates with high levels of immunosuppressive perfluorinated alkylates (Kielsen et al. 2016). Given the plans for global vaccination, once a vaccine is found, toxicologists may want to address such aspects as well.

In conclusion, there are many toxicological topics, which need clarification, and in particular, toxicological expertise can contribute in identifying risk factors and the underlying mechanisms, monitoring attempts to follow the spread of the disease, and possible threats to successful treatments either by drugs or by vaccines in the future.

\section{Compliance with ethical standards}

Conflict of interest The authors declare that they have no conflict of interest.

\section{References}

Boeckmans J, Rodrigues RM, Demuyser T, Pierard D, Vanhaecke T, Rogiers V (2020) COVID-19 and drug-induced liver injury: a problem of plenty or a petty point? Arch Toxicol 94(4):1367-1369

Fattorini D, Regoli F (2020) Role of the chronic air pollution levels in the Covid-19 outbreak risk in Italy. Environ Pollut 264:114732

Kielsen K, Shamim Z, Ryder LP et al (2016) Antibody response to booster vaccination with tetanus and diphtheria in adults exposed to perfluorinated alkylates. J Immunotoxicol 13(2):270-273

Yamada T, Horimoto H, Kameyama T et al (2016) Constitutive aryl hydrocarbon receptor signaling constrains type I interferon-mediated antiviral innate defense. Nat Immunol 17(6):687-694

Publisher's Note Springer Nature remains neutral with regard to jurisdictional claims in published maps and institutional affiliations. 